http://jmscr.igmpublication.org/home/

ISSN (e)-2347-176x ISSN (p) 2455-0450

crossref DOI: https://dx.doi.org/10.18535/jmscr/v7i10.149

Journal Of Medical Science And Clinical Research

IGM Publication

An Official Publication of IGM Publication

\title{
Development and Validation of the UV Spectrophotometric Method of Ursodeoxycholic Acid in Methanol
}

\author{
Authors \\ Asha Devi, Abhishek soni, Dr Amit Chaudhary \\ Abhilashi University, Mandi, Himachal Pardesh
}

\begin{abstract}
New developments have been taking place in the field of Development and validation of analytical method for the estimation of Ursodeoxychlic acid by using Uv spectroscopy. A simple, rapid, accurate and economical UV-spectroscopic method has been developed for the estimation of Ursodeoxycholic. The absorption maxima for Ursodeoxycholic acid is simulated liver fluid was found to be $232 \mathrm{~nm}$. The drug follows linearity in the concentration ranges $1-10 \mu \mathrm{g} / \mathrm{ml}$ at $232 \mathrm{~nm}$ with a correlation coefficient value of 0.998. The proposed method was applied to liver disease pharmaceutical formulation and \% drug estimated was found to be in the range of $99.99 \%$ that is good agreement with the lable claim. The accuracy of the method was checked by recovery experiment performed at three levels i.e., 80\%, 100\%, $120 \%$. The percent recovery was found to be in the range of 90-120\%. The low values of \% RSD are indicative of accuracy and reproducibility of method. The $\% R S D<2$ indicates that method is precise.

Keywords: UV-spectroscopy, Ursodeoxycholic acid, Method development, Accuracy, Precision.
\end{abstract}

\section{Introduction}

Ursodeoxycholic acid (UDCA) is a white, odourless, crystalline powder with a bitter taste. Chemically it is 3, 7-dihydroxy-5-cholan-24-oic acid. It is a water insoluble drug used as a drug for the dissolution of cholesterol gallstones because it reduces the cholesterol saturation of bile. The use of UDCA for the treatment of liver disease, such as primary biliary, biliary cirrhosis, chronic hepatitis and biliary pain has been demonstrated. However in vivo studies it shown the intestinal absorption and as a result the bioavailability of the drug are generally poor and unusual both among different subjects, and within the same subject. More than $50 \%$ is lost in the stool 9 after a single oral dose of $300 \mathrm{mg}$

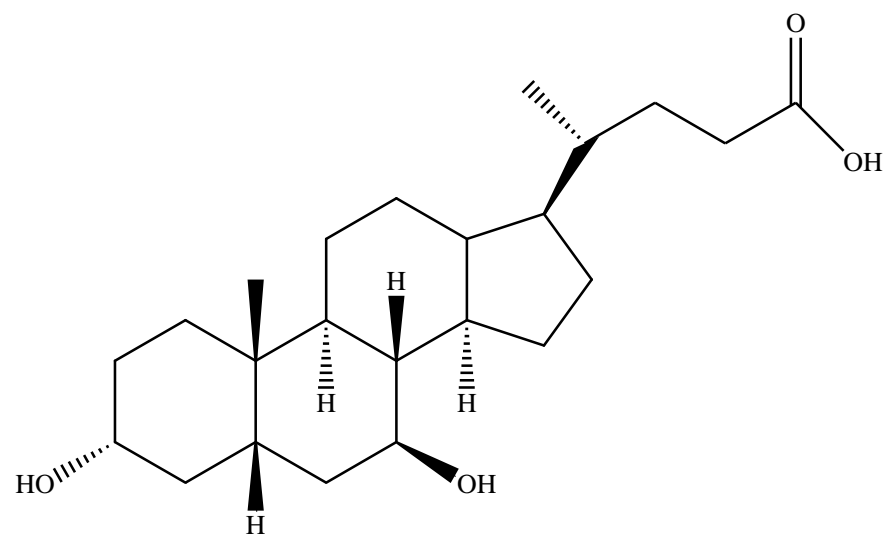

Fig.1 Ursodeoxycholic acids

\section{Materials and Methods}

\subsection{Materials}

Ursodeoxycholic acid standard powder was kindly supplied from Morepen Laoratories Limited, India. All chemical and reagent used were 
obtained from Research Laboratory, Abhilashi University and were of analytical grade.

\subsection{Preparation of standard stock solution}

Accurately weighed $10 \mathrm{mg}$ of Ursodeoxycholic acid was transferred to a $10 \mathrm{ml}$ volumetric flask, dissolve in $5 \mathrm{ml}$ simulated abdominal fluid by shaking and volume was adjusted with the same up to mark to give strength $1000 \mu \mathrm{g} / \mathrm{ml}$.

\subsection{Preparation of working standard}

From the above standard stock solution $5 \mathrm{ml}$ was further diluted to $50 \mathrm{ml}$ with SVF followed by sonication for 5-10 minute. The final strength was $100 \mu \mathrm{g} / \mathrm{ml}$. This stock was used to prepare various concentrations from $1-10 \mu \mathrm{g} / \mathrm{ml}$ by dilution with SVF.

\subsection{Selection of wavelength for analysis of Ursodeoxycholic acid}

Appropriate volume $1.2 \mathrm{ml}$ of working stock solution of Ursodeoxycholic acid was transferred into a $10 \mathrm{ml}$ volumetric flask, diluted with SVF up to the mark to give a concentration $12 \mu \mathrm{g} / \mathrm{ml}$. The resulting solution was scanned between 200-400 $\mathrm{nm}$ using SVF as blank. The spectrum showed the absorbance maxims at $232 \mathrm{~nm}$. This maxima was further used to get calibration curve.

\section{Validation of Method}

Validation is a process of establishing documented evidence, which provides a high degree of assurance that a specific activity will consistently produce a desired result, or a product meeting was validated for different parameter like Linearity, Accuracy, Precision, Specificity, Robustness, Limit of detection (LOD), and Limit of Quantification (LOQ).

\subsection{Linearity study}

Stock solutions of Ursodeoxycholic acid (1, 2, 3, $4,5,6,7,8,9$, and $10 \mathrm{ml}$ ) were pipetted, into a series of ten $50 \mathrm{ml}$ volumetric flask. The volume in each volumetric flask was made up to the mark with $50 \% \mathrm{v} / \mathrm{v}$ aqueous methanol and the content was mixed so as to obtain a final concentration in the range of 2 to $20 \mu \mathrm{g} / \mathrm{ml}$. The absorbance of the solution is measured at $232 \mathrm{~nm}$ against $50 \% \mathrm{v} / \mathrm{v}$ aqueous methanol used as a blank.

\subsection{Accuracy}

To the pre analysed sample solution, a known amount of standard stock solution at three different levels (80,100 and $120 \%)$. The sample was reanalysed by the proposed method. The solutions were prepared in triplicate and the accuracy was indicated in \%.

\subsection{Precision}

The term precision id defined by the ISO International Vocabulary of Basic and General Terms in Metrology (ISO-VIM) and ICH as the closeness of agreement between quantity values obtained by replicate measurement of a quantity under specific condition. Assessing the precision implies expressing numerically the random error or the degree of dispersion of a set of individual measurements by means of the standard deviation, the variance, or the coefficient of variation.

\subsubsection{Precision of Repeatability}

It is the concordance of a series of measurements of the same quantity when the experiments are conducted under same condition (analyst, apparatus, instrument, and day) in a rapid succession. For this experiment, standard solution of Ursodeoxycholic acid at $290(10 \mu \mathrm{g} / \mathrm{ml})$ was prepared and analysed six times as per the proposed method.

\subsubsection{Intermediate Precision}

It is the concordance of a series of measurement of the same quantity when the experiments are conducted within the same laboratory under different condition (analyte, apparatus, instrument, and day). Standard solution of Ursodeoxycholic acid at $290(10 \mu \mathrm{g} / \mathrm{ml})$ was prepared and analyzed as per the proposed method

\subsection{Sensitivity}

The sensitivity of the measurement of Ursodeoxycholic acid was estimated in the term of the limit of detection (LOD) and limit of quantification (LOQ) by aid of proposed method. The LOD and LOQ of the proposed method were determined by using the equation:

$$
\mathrm{LOD}=3.3 \sigma / \mathrm{S}, \quad \mathrm{LOQ}=10 \sigma / \mathrm{S}
$$

Where $\sigma$ is the standard deviation of the response (Y intercept) and $\mathrm{S}$ is the slop of the calibration curve. 


\subsection{System suitability}

A system suitability test of the spectrophotometric system was performed before each validation run. Six replicate reading of standard preparation were taken and \% RSD of standard reading were taken for same. Acceptance criteria foe system suitability, \% RSD of standard reading not more than $2.0 \%$, were full fill during all validation parameter.

\section{Conclusion}

The present analytical method was validated as pet ICH Q2 (R1) guideline and it meets to specific acceptance criteria. It is concluded that the analytical method was specific, precision, linear, and accurate while estimating the commercial formulation without interference of the excipients and other additive. Hence the present analytical method can be used for the routine determination of Ursodeoxycholic acid in pure pharmaceutical formulation at the minimum cost.

\section{References}

1. Fromn H. Gallstone dissolution and the cholesterol-bile acid-lipoprotien axis: propertious effect of Ursodeoxycholic acid. Gastroenterology 1984; 87: 229-233.

2. Ward A, Brogden RN, Heel RC. Ursodeoxycholic acid: A review of its p'cological properties and therapeutic efficacy. Drug 1984; 27-95

3. ICHQ, 2B, Validation of analytical procedure. Definition and terminology. CPMIII/5626/94. March 1995; Geneva, Switzerland.

4. ICH, 2B, Validation of analytical procedure. Methodology (CPMP/95) Nov. 1996. Geneva, Switzerland

5. Beuers U, Boyer JL, Paumgartner G. Ursodeoxycholic acid in cholestasis: potential mechanism of action and therapeutic application. Hepatology 1998; 28: 1449-53.
6. Hagey LR, et al. Ursodeoxycholic acid in the Ursidae: biliary bile acids of bears, pandas, and related carnivores. J Lipid Res 1993; 34: 1911-19.

7. Akare $\mathrm{S}$, et al. Ursodeoxycholic acid modulates histone acetylation and induces differentitation and senescence. International Journal of Cancer. Journal International Du Cancer 119 (12): 29582969.

8. Amaral JD, et al. Bile acids regulation of apoptosis by Ursodeoxycholic acid Journal of Lipid Research 2009; 50 (9): 17211734.

9. Tait N, Little JM. The treatment of gall stone. BMJ 1995; 3(11): 99-105.

10. Portincasa $\mathrm{P}$, et al. Medical treatment of cholesterol gallstone: old, current and new perspectives. Curr Med chem. 2009; 16: 1531-1542. 